Objectives Neisseria gonorrhoeae (NG) and Chlamydia trachomatis (CT) are sexually transmitted infections (STIs). Most infections are asymptomatic, representing an important reservoir for transmission. Without treatment, complications such as infertility may occur. Moreover, $33 \%$ of CT/NG co-infection rate has been reported. Since 2010, combined screening of CT and NG by PCR in asymptomatic population has been recommended by the French Health Authority. Thus, the aim of this study was to assess the interest of the new Cepheid Xpert ${ }^{\circledR}$ CT/NG Assay, a real-time PCR test for the automated and rapid detection and differentiation of CT and NG genomic DNA, in population with systematically screening such as induced abortion.

Methods Between July and November 2012, 634 urogenital samples were received in our laboratory to detect CT and/or NG infections with the Xpert CT/NG assay.

Results Of the 634 samples included in this study, 61 (9.6\%) were CT positive, 19 (2.9\%) were NG positive. Among the $61 \mathrm{CT}$ positives, $10(1.6 \%)$ were positive for both CT and NG. Concerning the 177 samples performed in case of induced abortion, 27 (15.3\%) were CT positive, $9(5.1 \%)$ were NG positive and 5 (2.8\%) were positive for both pathogens.

Conclusion The results revealed a global prevalence $(9.6 \%)$ of CT infections, this percentage being higher in women screened for induced abortion. Although, many clinicians tend to only request testing for CT, our results demonstrate the value of the detection of both CT and NG by Xpert CT/NG. This new test allows a more rapid, accurate detection and optimises the management of STIs by clinicians. Finally, the screening of asymptomatic population helps also to reduce the transmission and is a more cost effectiveness alternative in screening settings.

\section{P2.012 CLINICAL CARE PATHWAYS USING CHLAMYDIA AND GONORRHOEA TESTS ARE EVOLVING: POINT OF CARE NUCLEIC ACID AMPLIFICATION TESTS MAY REDUCE GENITOURINARY MEDICINE SERVICE DELIVERY COSTS}

doi:10.1136/sextrans-2013-051184.0277

1.2E J Adams, 'A Ehrlich, ${ }^{2} \mathrm{~K}$ M E Turner, ${ }^{1} \mathrm{~K}$ Shah, ${ }^{2} \mathrm{~J}$ Macleod, ${ }^{3} \mathrm{~S}$ Goldenberg, ${ }^{4} \mathrm{~V}$ Pearce, ${ }^{2 P} \mathrm{~J}$ Horner. 'Aquarius Population Health, London, UK; ${ }^{2}$ University of Bristol, Bristol, UK; ${ }^{3}$ Guy's \& St. Thomas' NHS Foundation Trust and King's College, London, UK; ${ }^{4} \mathrm{~V}$ Pearce Consulting Ltd, Hove, UK

Background We aimed to explore new patient pathways using a chlamydia/gonorrhoea (CT/NG) point of care nucleic acid amplification tests (POC NAAT), and estimate and compare the costs of the new pathways to the current pathways using standard laboratory-based NAAT testing.

Methods A qualitative and quantitative approach was used. Focus groups were conducted with four sexual health clinics in the UK. They mapped out current pathways in which a CT/NG test was used, and then constructed new pathways using a POC NAAT. These pathways were then costed using a model built in Excel, and the cost of the current and POC NAAT pathways compared.

Results Pathways using a POC NAAT for asymptomatic and symptomatic patients and CT/NG only tests were shorter and less expensive than most of the current pathways (average savings of $£ 6-8$ per pathway if the POCT costs £18 per test). Clinicians identified several potential benefits to introducing the test including faster time to treatment, more accurate diagnosis of symptomatic patients, and therefore less syndromic management, which is likely to result in better care for patients. Several theoretical risks and limitations were identified in the workshops although these were not assessed in the study.

Conclusion A point of care test could be introduced to services and reduce current costs, and may mean more appropriate and quicker care for positive patients.

\section{P2.013 LYMPHOGRANULOMA VENEREUM CASES IDENTIFIED IN PATIENTS ATTENDING A STD OUTPATIENTS CLINIC IN ITALY}

doi:10.1136/sextrans-2013-051184.0278

A Marangoni, ${ }^{2} \mathrm{~A}$ D'Antuono, ${ }^{2} \mathrm{~A}$ Filippini, ${ }^{2} \mathrm{~S}$ Bellavista, ${ }^{2} \mathrm{C}$ Baraldi, ${ }^{1} \mathrm{C}$ Foschi, ${ }^{1} \mathrm{P}$ Nardini, 'M Compri, 'R Cevenini. 'University of Bologna, Microbiology, DIMES, Bologna, Italy; 'University of Bologna, Dermatology, DIMES, Bologna, Italy

Background Lymphogranuloma venereum (LGV) is a systemic sexually transmitted infection caused by Chlamydia trachomatis (CT) serovars L1-L3. In the recent outbreaks the classic clinical presentation with inguinal syndrome is giving way to anorectal primitive syndrome in men having sex with men (MSM). Here we report about 6 cases of LGV identified during 2012.

Methods A prospective study was performed with 78 rectal specimens obtained from MSM attending the STD Outpatients Clinic of S. Orsola Hospital, Bologna. All the patients were enrolled because having unsafe receptive anal sex intercourses. Samples were tested by Versant CT/GC DNA 1.0 (Siemens). Genotyping was performed with RFLP method for ompl gene, using AluI and DdeI as restriction enzymes. Results We found a total of 11 rectal swabs positive for CT. RFLP analysis showed 6 L2 genotypes and 5 non-LGV genotypes (3 were $\mathrm{E}$, and the others $\mathrm{H}$ and $\mathrm{J}$ ).

The five non-LGV infected patients showed no symptoms. On the contrary, at the enrollment perianal ulcers, proctitis and painful lymphadenopathy were found in three LGV cases, whereas perianal ulcers and proctitis in the remaining three ones.

Before the correct diagnosis the patients had been investigated for several months for a broad range of other conditions, including traumatic warts, and/or gastroenteric syndromes. Three patients had undergone endoscopic procedures and ultrasound scans.

All the LGV cases presented at least one more sexually transmitted infection. Treatment with doxycycline $(100 \mathrm{mg}$ b.i.d. for 21 days) was successful. At control, case 1 had a positive result for Neisseria gonorrhoeae in his rectal swab, thus demonstrating his high risk sexual behaviour.

Conclusion A firm diagnosis and early treatment of LGV can prevent the development of serious sequelae. Since the ulcerative nature of LGV may facilitate transmission and acquisition of other STDs, enhanced surveillance systems and strengthened case ascertainment would be desirable.

\section{P2.014 DEVELOPMENT AND ANALYTICAL PERFORMANCE OF NEW CT/NG NUCLEIC ACID AMPLIFIED TEST (NAAT)}

doi:10.1136/sextrans-2013-051184.0279

F A Weir, P Foxall, S Alugupally, M Satya, K Ho, A Lee. Cepheid, Sunnyvale, CA, United States

Background Moderately complex NAATs that can provide fast, actionable results could reduce the burden of Chlamydia trachomatis (CT) and Neisseria gonorrhoeae (NG) infections. The Xpert ${ }^{\circledR}$ CT/NG Assay is a recently FDA-cleared, moderately complex assay for the detection of CT and NG in patient collected vaginal swabs, endocervical swabs, and male and female urines with a turnaround time of 87 minutes.

Methods Primers and probes were designed against specific CT and NG candidate genomic targets and screened for exclusivity with genomic DNA extracted from non-trachomal Chlamydia/Chlamydophila and non-gonococcal Neisseria species. For inclusivity, genomic DNA extracted from the 15 CT serovars and 230 geographically diverse NG strains was used. A multiplex assay, running on the GeneXpert platform, was designed to detect one CT target, two independent unique NG targets and two control targets. Analytical reactivity and specificity were determined by testing whole 